\title{
Jason P. Sheehan and Peter C. Gerszten: Controversies in stereotactic radiosurgery. Best evidence recommendations
}

\author{
Thieme Publishers, New York, Stuttgart. 2014, 270 pp, 130 illustrations, Hardback. \\ ISBN: 9781604068412, eBook - Available from ebookstore.thieme.com, Amazon, Barnes \\ \& Noble, and iTunes, eISBN: 9781604068429, $€ 109.99 / \$ 129.99$
}

\author{
Duparc Fabrice · Stéphane Derrey
}

Published online: 12 August 2014

(C) Springer-Verlag France 2014

Since the introduction of radiosurgery therapy over 60 years ago, radiosurgical tools, neuro-imaging and the understanding of how radiosurgery works have considerably progressed. By increasing the accuracy and precision of radiosurgical procedures, which aim to mimic a surgical resection, new clinical applications have emerged inside the cranium but also in the spinal cord.

In Controversies in Stereotactic radiosurgery, the authors JP Sheehan and PC Gerszten have combined the experiences of the most experiment teams in the different field of radiosurgical applications. Jason Sheehan is Professor of Neurological Surgery, University of Virginia, Charlottesville, and Peter Carlos Gerszten, is Professor of Neurological Surgery and Radiation Oncology, University of Pittsburgh Medical Center, Pennsylvania, USA.

In a well-illustrated book, the authors clearly exposed the results, safety and limits of radiological treatment without omitting microsurgical approach and conventional radiotherapy options. The management of cranial benign and malignant tumor, vascular malformation, functional indications such as trigeminal neuralgia and spinal lesions is discussed with the goal to purpose to the patient the best strategy.
Interestingly, because radiosurgery is able to deliver high dose of radiation to the target, authors remind us the absolute necessity to exactly define the surrounding structures nearby the irradiated volume. It is of note that the knowledge of morphological, functional and radiological anatomy is required to plan a safe radiosurgical procedure. For example, special chapter has been dedicated to the optic apparatus as a challenge to respect in some indications. Similar anatomical considerations are evoked for lesion located within or in the vicinity of the brainstem or the spinal cord with the aim to systematically exclude the sensitive neural tissue surrounding the target.

In this book, new lights and controversies about radiosurgical treatments are given to the readers. Controversies in Stereotactic radiosurgery should bring to the interest of neurosurgeons, radiosurgeons, neurooncologist and radiotherapist as it covers a very large panel of radiosurgical indications on the basis of the most relevant clinical studies published on each topic.

S. Derrey, MD-PhD, Neurosurgeon.

D. Fabrice $(\bowtie)$

Laboratory of Anatomy, Rouen University, 22 Bld Gambetta,

76183 Rouen Cedex 1, France

e-mail: fabrice.duparc@univ-rouen.fr

S. Derrey

Department of Neurosurgery, Rouen University Hospital,

Bld Gambetta, 76031 Rouen Cedex, France 